Case Report

\title{
Challenges of Diagnosing Severe Ehrlichiosis in Orthotopic Liver Transplant Recipients
}

\author{
Melissa Parkinson (D, ${ }^{1}$ Spandana Vuyyuru, ${ }^{2}$ Jay Patel, $^{2}$ and Chinelo Animalu ${ }^{3}$ \\ ${ }^{1}$ Internal Medicine and Pediatrics Residency Program, University of Tennessee Health Science Center, Memphis, USA \\ ${ }^{2}$ College of Medicine, University of Tennessee Health Science Center, Memphis, USA \\ ${ }^{3}$ Division of Infectious Diseases, University of Tennessee Health Science Center, Memphis, USA
}

Correspondence should be addressed to Melissa Parkinson; mparkin1@uthsc.edu

Received 28 May 2021; Accepted 15 October 2021; Published 17 November 2021

Academic Editor: Ryszard Grenda

Copyright (c) 2021 Melissa Parkinson et al. This is an open access article distributed under the Creative Commons Attribution License, which permits unrestricted use, distribution, and reproduction in any medium, provided the original work is properly cited.

\begin{abstract}
In recent solid organ transplant recipients, acute febrile illness is usually a source of grave concern and a diagnostic dilemma, especially if no response is noted after initiation of broad antimicrobial therapy. Human Monocytic Ehrlichiosis (HME) is a tick-borne illness caused by Ehrlichia chaffeensis and is not considered an opportunistic infection in immunocompromised patients such as solid organ transplant patients. Ehrlichiosis in immunocompromised patients can be life-threatening, and a strong index of suspicion is needed, especially in patients who live in endemic areas, for proper treatment initiation with doxycycline. We report a case of a 40-year-old male who received an orthotopic liver transplant six months earlier secondary to primary sclerosing cholangitis, on chronic immunosuppressive medication, who presented with complaints of sudden onset fever associated with nausea, vomiting, and diarrhea. Initial extensive infectious workup was negative and no response to empiric antimicrobials. There was suspicion for ehrlichiosis prompting empiric doxycycline use. Subsequently, E. chaffeensis polymerase chain reaction (PCR) was positive, and the antibiotic regimen was de-escalated to only doxycycline with complete resolution of his symptoms and progressive improvement in previously abnormal biochemical indices.
\end{abstract}

\section{Background and Introduction}

Solid organ transplant is a life-saving treatment for many patients with end-stage organ disease. However, the immunosuppressive regimens these patients require leave them susceptible to severe infections from both opportunistic and common organisms. The wide range of potential infectious agents and blunted immune response in these patients can make prompt diagnosis and treatment difficult.

Ehrlichiosis is a disease caused by obligate intracellular bacteria from the genus Ehrlichia transmitted via tick bite that infects human and animal leukocytes. E. chaffeensis is the most common species to infect humans and causes Human Monocytic Ehrlichiosis (HME). Rates of reported HME rose from just 200 in 2000 to 1,799 in 2018 and continue to rise, making it an increasingly important illness [1]. E. chaffeensis is transmitted primarily through the lone star tick and is endemic (Figure 1 [1]) to the southeastern, south-central, and mid-Atlantic United States [1]. Contact with the ticks and subsequent transmission can occur in areas where humans are with natural tick reservoirs (e.g., cats and deer) (Figure 2 [2]).

Manifestation of HME can range from asymptomatic to severe, with severe illness being more likely in the elderly, those with comorbid conditions, and immunocompromised patients. Many of the common early symptoms are nonspecific, including fever, chills, malaise, myalgia, headache, nausea, vomiting, diarrhea, and loss of appetite. Rash and neurological symptoms like confusion may also be present [1]. Delays in treatment and immunocompromised states can lead to more severe late disease with meningoencephalitis, respiratory failure, uncontrolled bleeding, organ failure, and death [1]. Definitive diagnosis can be made with several laboratory techniques, including serology, polymerase chain 


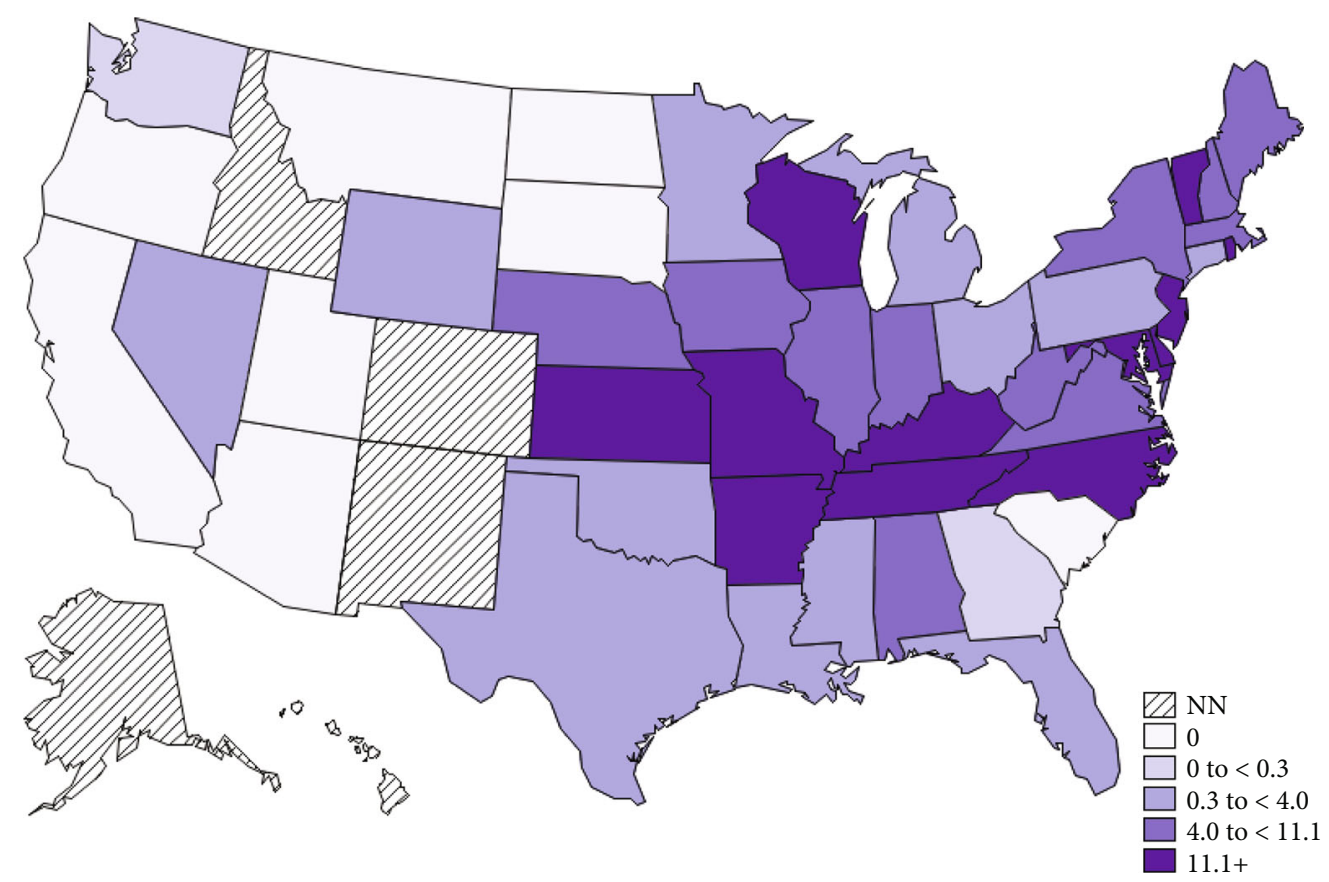

Figure 1: Annual incidence (per million population) of HME in the United States in 2019. This graph shows the general distribution and incidence of HME in the United States, with most cases occurring in the south-central and southeastern United States. Courtesy of the Centers for Disease Control and Prevention [1].

reaction (PCR), and buffy coat examination. Treatment with doxycycline is usually initiated based on a presumptive diagnosis when clinical suspicion is high. However, when tickborne illness is not suspected early on, definitive treatment can be critically delayed due to the initial empiric antibiotic regimen's exclusion of E. chaffeensis coverage.

\section{Case Report}

We present the case of a 40 -year-old male who six months prior underwent an orthotopic liver transplant secondary to primary sclerosing cholangitis on chronic immunosuppressive therapy. He presented to the emergency room with complaints of sudden onset fever ongoing for three days associated with nausea, vomiting, and diarrhea (about five to six watery bowel movements per day). He also endorsed fatigue. He denied chest pain, shortness of breath, headache, confusion, or vision changes. His immunosuppressive medications consisted of prednisone, mycophenolate mofetil, tacrolimus, and valganciclovir. He previously had cytomegalovirus (CMV) viremia, for which he was treated with valganciclovir. Serial CMV PCR was negative, and the valganciclovir dose was decreased to a maintenance dose.

At the time of initial presentation, he was febrile with a maximum temperature of $38.9^{\circ} \mathrm{C}$, blood pressure 93/ $55 \mathrm{mmHg}$, pulse 101 beats/min, and oxygen saturation $98 \%$ on room air. He was alert, oriented, cooperative, and in no apparent distress. The physical exam was unremarkable, with no rashes or lesions noted.

Initial labs revealed sodium of $130 \mathrm{mEq} / \mathrm{L}$, potassium $5.6 \mathrm{mEq} / \mathrm{L}$, chloride $105 \mathrm{mEq} / \mathrm{L}$, creatinine $1.60 \mathrm{mg} / \mathrm{dL}$, albu- $\min 4.6 \mathrm{~g} / \mathrm{dL}$, total bilirubin $1.2 \mathrm{mg} / \mathrm{dL}$, AST $44 \mathrm{U} / \mathrm{L}$, ALT $51 \mathrm{U} / \mathrm{L}, \mathrm{WBC} 1.70$ thou/mcL with absolute neutrophil count of 0.6 thou $/ \mathrm{mcL}$, platelets 40 thou $/ \mathrm{mcL}$, and hemoglobin $8.7 \mathrm{~g} / \mathrm{dL}$. CMV PCR was negative. Blood cultures were sent and were subsequently negative. A chest X-ray did not reveal any acute abnormalities. Abdominal ultrasound showed findings consistent with medical renal disease only. Gastrointestinal PCR panel was negative for enterotoxigenic E. coli, Giardia, Norovirus, Salmonella, Shiga-like Toxin producing E. coli, Plesiomonas shigelloides, Vibrio, Yersinia enterocolitica, enteroaggregative E. coli, Cyclospora cayetanensis, Entamoeba histolytica, Adenovirus F 40/41, Astrovirus, Sapovirus, Enteropathogenic E. coli, and Shigella/ Enteroinvasive E. coli. Clostridium difficile and SARS-CoV2 PCR were negative. Serum Cryptococcus neoformans and histoplasmosis antigens were negative. Acute hepatitis A, B, and $\mathrm{C}$ panel was negative. Rocky Mountain spotted fever IgM titer and Rickettsia typhi IgM and IgG antibodies were negative. Urinalysis was unremarkable.

He was started on empiric vancomycin, meropenem, and micafungin (doses adjusted for renal function) without any clinical improvement in 48 hours. His immunosuppressive medication doses were reduced appropriately. His fever persisted, and renal function, as well as liver functions, declined rapidly (Figure 3), prompting an infectious disease consult. On further questioning, the patient revealed he worked as a resource manager in a state park and is exposed regularly to ticks; he did not recall any obvious tick bites and had never had to pluck out a tick from his skin. He denied eating unpasteurized goods and did not consume uncooked meat or fish. He had not been swimming in public pools. 


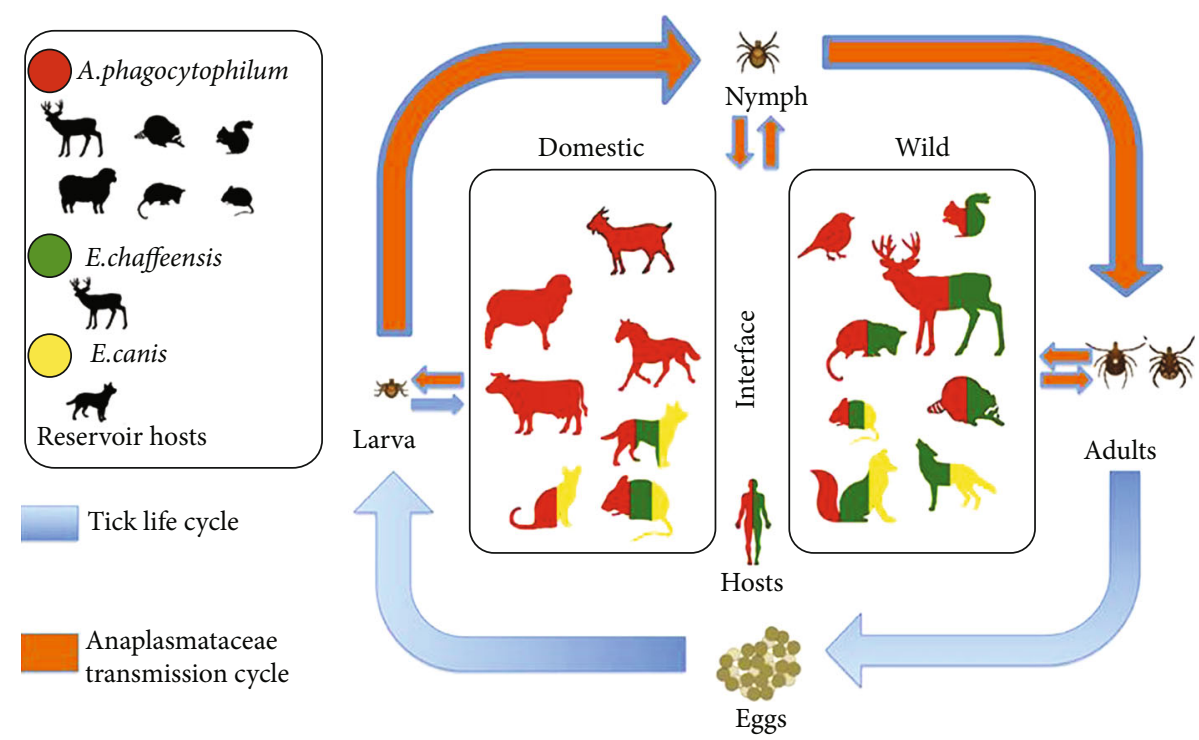

Figure 2: Tick-borne pathogen transmission. This image shows the life cycle of Ehrlichia species and which animal different tick-borne pathogens can inhabit. This also shows the different ways humans can come in contact with ticks. Image courtesy of the open access article by Rojero-Vázquez et al. [2].

Given this history, intravenous doxycycline for presumptive ehrlichiosis was initiated on hospital day 3 while awaiting studies. Subsequently, E. chaffeensis PCR returned positive. He required a short ICU stay for vasopressor support due to septic shock. Given his severe acute kidney injury and creatinine clearance decline to $<15 \mathrm{~mL} / \mathrm{min}$, hemodialysis was initiated on hospital day 4 . His immunosuppressive medication regimen was initially held on admission and later changed to prednisone and tacrolimus, while mycophenolate was discontinued.

After initiation of doxycycline, clinical improvement was noted both in symptoms and laboratory indices (Figure 3). He completed a 10-day course of doxycycline and was taken off hemodialysis on hospital day 10 . The patient was discharged home in a stable condition and, since his discharge, has continued to do well.

\section{Discussion}

Since its first recognition in the late 1980s, cases of HME have been rising, with over 1000 cases reported by the CDC annually since 2012, occurring in both immunocompromised and immunocompetent patients [1]. Case numbers in 2018 were over eight times higher than in 2000, making HME important to consider when a patient presents with nonspecific findings, particularly in an endemic area [1]. While many studies examine HME in the context of the United States, there has been an increasing incidence of HME internationally on continents such as Asia and Africa [3]. Various studies are emerging that have shown Ehrlichia species found in different animal vectors that were thought not to host them, suggesting that there could be more unknown reservoirs of this bacterium and that overall distribution and disease of this bacterium may be more widespread than previously thought [4-6].

The number of HME cases occurring in solid-organ transplant (SOT) recipients makes up a small but significant portion of overall cases. It is important to recognize this as a potential cause of infection in SOT patients, particularly in endemic areas. A literature search was done, and all known cases of Ehrlichia complicating various SOT are tabulated in Table 1. There were 143 found in the literature, and, interestingly, doxycycline was used in the vast majority of these patients, and most patients survived as well.

Diagnosis of ehrlichiosis in transplant recipients can often be challenging due to the low prevalence of solidorgan transplant (SOT) HME, nonspecific symptoms, which can be seen in a wide range of infection as well as organ rejection, and blunting of the immune response [3, 4]. Common symptoms reported from Ehrlichia infections in transplant patients from one review of cases include a history of fatigue (30\%), subjective fevers (95-100\%), headache (55-60\%), and GI symptoms (40-60\%), which were similar across other reports [7-11]. Our patient presented with fever, diarrhea, nausea, vomiting, and fatigue-similar symptoms to those reported in other SOT patients; however, he had no headache or other neurologic symptoms. Less commonly, HME can present with cutaneous manifestations, pulmonary embolism, severe disease leading to hemophagocytic lymphohistiocytosis, and fulminant organ failure [12].

Preemptive treatment is usually initiated for suspected ehrlichiosis while workup for definitive diagnosis is in progress. Several current diagnostic methods include serologies, peripheral blood smear (buffy-coat preparation) examination, PCR, immunohistochemical (IHC) staining, and culture. Serologic studies can be done via an indirect fluorescent antibody test (IFA) or enzyme-linked immunosorbent 


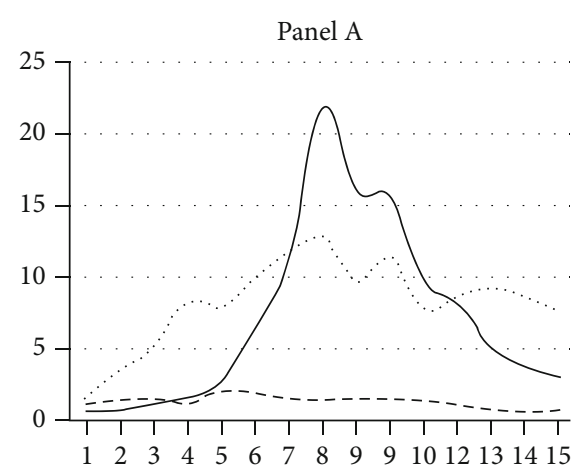

Hospital day

$$
\begin{aligned}
& \text {..... Creatinine }(\mathrm{mg} / \mathrm{dL}) \\
& \ldots \text {-.. Total bilirubin }(\mathrm{mg} / \mathrm{dL}) \\
& \ldots \text { WBC }(\text { thou/dL) }
\end{aligned}
$$

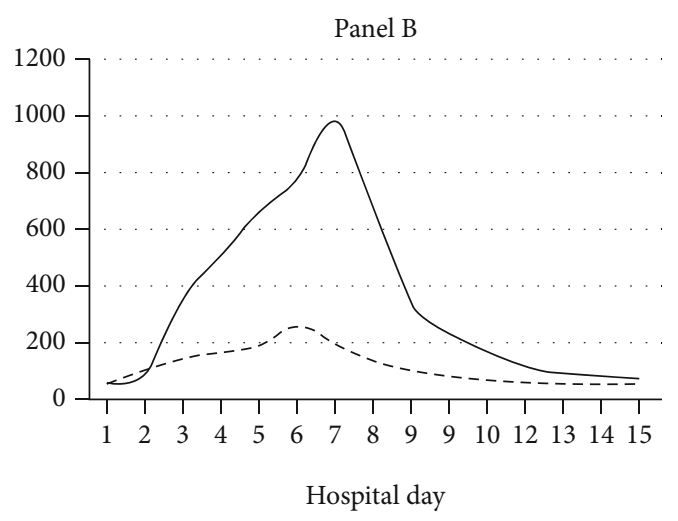

$-\operatorname{AST}(\mathrm{U} / \mathrm{L})$

-.- $\operatorname{ALT}(\mathrm{U} / \mathrm{L})$

Figure 3: Trends of key lab findings throughout hospital stay. Doxycycline was started on hospital day 3 for suspected ehrlichiosis. The patient had been started on hemodialysis due to acute renal failure on hospital day 4 and was taken off on day 10 due to marked clinical improvement. The progression of creatinine $(\mathrm{mg} / \mathrm{dL})$, WBC count (thou/mcL), and total bilirubin $(\mathrm{mg} / \mathrm{dL})$ over his hospital stay is seen in (a). The progression of AST (U/L) and ALT (U/L) over his hospital stay is seen in (b).

assay (ELISA). IFA is the standard reference test for ehrlichiosis; it is widely available and can be obtained through state health departments [13]. This assay requires comparing antibody titers of acute and convalescent serum samples collected 2-4 weeks apart to demonstrate evidence of a fourfold seroconversion. While the test is $94-100 \%$ sensitive, antibody titers are usually low during the first week of illness, decreasing utility in detecting HME in the early phase [13-15]. Thus, IFA is infrequently due to minimal early clinical utility and time-consuming nature [16]. In addition, ELISA also measures antibody titers and is excellent for confirming findings from IFA, but it is not very good at evaluating variations in antibody titers with time $[13,16]$. Thus, similar to IFA, ELISA's clinical utility is low and better suited for clinical trials [16]. Peripheral blood smear examination using a buffy-coat preparation is usually useful within the first week of illness, where microscopic examination may reveal a collection of microcolonies of Ehrlichia in the cytoplasm of white blood cells [13]. The utility of examining peripheral blood depends on the patient population [17]. Sensitivity of doing a peripheral blood smear in immunocompetent people is $17 \%$; however, immunocompromised patients can have a sensitivity as high as $100 \%$, increasing utility in the transplant population $[17,18]$. This is likely due to the immunocompromised state enabling a more considerable morula burden, increasing the chance of seeing morula on slides. PCR is a rapid diagnostic test that is becoming more widely available, and it has good sensitivity $(85 \%)$ and specificity $(100 \%)[14,19]$. It is most sensitive within the first week of symptom onset, and a positive result is considered diagnostic; however, a negative result does not rule out ehrlichiosis, especially if antibiotics have begun [20]. IHC staining of ehrlichial antigens in tissue can be done, but this is not widely available and not routinely used for acute diagnosis, limiting clinical utility [20]. These can be performed in bone marrow, live tissue specimens, or postmortem specimens [20]. Lastly, culture is slow, not widely available, and is not very useful $[16,20]$.

This case highlights the importance of taking a thorough history to identify potential exposures. Treatment for HME was only initiated after a thorough exposure history revealed that the patient worked outside and had significant tick exposure. Doxycycline is the drug of choice for the treatment of HME [21]. However, in some patients with a severe allergy to doxycycline, alternative therapy such as rifampin and chloramphenicol has been used even though there are no robust studies available at this time supporting the routine use of these agents [22]. There are case reports where successful use of these agents has been documented; chloramphenicol is not readily available in the United States and is associated with hematological side effects [22]. Travel history to endemic areas, work and hobby history, and a thorough skin exam should all be performed when concerned for possible HME infection. While a definitive diagnosis of HME can be made using a variety of methods, including PCR and serology, there are diagnostic clues that can be identified in an initial lab workup that is typical of HME, including leukopenia, thrombocytopenia, elevated liver enzymes, elevated creatinine, and less commonly hyponatremia, pancytopenia, and decreased serum albumin levels [7-11]. In this case, the patient had lab findings consistent with HME, including hyponatremia, pancytopenia, elevated creatinine, and elevated liver enzymes (Figure 3).

Early detection of disease is crucial to prevent disease progression and end-organ damage. In one study, delayed time to treatment was associated with the need for ICU admission [8]. In this case, delays in the patient's diagnosis led to renal failure requiring hemodialysis and septic shock requiring vasopressor support. Immunosuppression has often been suggested as a possible risk factor for worse prognosis and ICU admission, but the data are mixed. One study found that immunosuppression was associated with decreased need for ICU admission, while others showed that 
TABLE 1: All cases of ehrlichiosis complicating solid organ transplants.

\begin{tabular}{|c|c|c|c|c|c|c|c|}
\hline Total cases & $\begin{array}{c}\text { Age median } \\
\text { (Yr, range) }\end{array}$ & Sex & Transplant type & Diagnosis method & Management & Outcome & References \\
\hline 25 & $54(26-68)$ & $68 \% \mathrm{M}$ & $\mathrm{H}(2), \mathrm{Li}(5), \mathrm{Lu}(5), \mathrm{R}(13)$ & PCR & Dox & $S$ & Lawrence et al. [10] \\
\hline 51 & $57(9-72)$ & $75 \% \mathrm{M}$ & $\begin{array}{l}\mathrm{H}(12), \mathrm{Li}(7), \mathrm{Lu}(12) \\
\text { R (18), R/P (2) }\end{array}$ & PCR & NA & $S$ & Otrock et al. [11] \\
\hline 1 & 69 & $\mathrm{~F}$ & $\mathrm{Lu}$ & PCR, PBS & Dox & $S$ & Regunath et al. [18] \\
\hline 15 & $50(15-73)$ & $87 \% \mathrm{M}$ & $\mathrm{H}(6), \mathrm{Li}(1), \mathrm{Lu}(1), \mathrm{R}(7)$ & $\begin{array}{c}\text { PCR }(93 \%), \\
\text { Ser }(7 \%)\end{array}$ & Dox & S & Thomas et al.[9] \\
\hline 2 & 56,57 & $50 \% \mathrm{M}$ & $\mathrm{R}(2)$ & $\begin{array}{l}\text { PCR (50\%), } \\
\text { Ser }(50 \%)\end{array}$ & Dox, Tigecycline & $S$ & Sachdev et al. [25] \\
\hline 1 & 38 & M & $\mathrm{Lu}$ & PCR, PBS & Dox & S & Safdar et al. [23] \\
\hline 1 & 57 & M & $\mathrm{Li}$ & PCR, Ser & Dox & S & Liddell et al. [24] \\
\hline 1 & 63 & M & $\mathrm{R}$ & PCR, PBS & Dox & S & Kumar et al. [26] \\
\hline 1 & 60 & M & $\mathrm{R}$ & PCR, PBS, Ser & Dox & S & Cotant et al. [27] \\
\hline 1 & 11 & M & $\mathrm{R}$ & PCR & Dox & $S$ & Buller et al. [28] \\
\hline 1 & NA & M & $\mathrm{R}$ & PCR & Dox & S & Sadikot et al. [29] \\
\hline 1 & 35 & M & $\mathrm{R}$ & Ser, PCR & Dox & $S$ & Schutze et al. [30] \\
\hline 1 & 47 & M & $\mathrm{Li}$ & PCR & Dox & S & Tan et al. [14] \\
\hline 1 & 27 & M & $\mathrm{R}$ & PBS, Ser & Dox & S & Dorn et al. [31] \\
\hline 1 & 35 & $\mathrm{~F}$ & $\mathrm{R}$ & PCR & Dox & $S$ & Masterson et al. [7] \\
\hline 27 & NA & NA & NA & PCR & Dox & NA & Kuriakose et al. [8] \\
\hline 1 & 51 & M & $\mathrm{Li}$ & Ser & Dox & S & Antony et al. [32] \\
\hline 3 & $38,41,50$ & $66 \% \mathrm{M}$ & $\mathrm{P} / \mathrm{R}, \mathrm{P} / \mathrm{R}, \mathrm{P}$ & PBS & Dox & $66 \% \mathrm{~S}$ & Trofe et al. [33] \\
\hline 1 & 67 & M & $\mathrm{R}$ & PBS, PCR & Dox & S & Adachi et al. [34] \\
\hline 1 & 66 & $\mathrm{~F}$ & $\mathrm{R}$ & PBS, PCR & Dox & S & Vannorsdall et al. [35] \\
\hline 1 & 57 & M & $\mathrm{R}$ & PCR & Dox & S & Hassan et al. [36] \\
\hline 5 & $43(5-70)$ & M & Lu (1), R (4) & $\begin{array}{l}\text { PCR (80\%); } \\
\text { PBS (20\%) }\end{array}$ & Dox & $60 \% \mathrm{~S}$ & Saha et al. [15] \\
\hline
\end{tabular}

D: death; Dox: doxycycline; F: female; H: heart; Li: liver; Lu: lung; M: male; NA: not available; P: pancreas; PBS: peripheral blood smear; PCR: polymerase chain reaction; R: renal; Ser: serology; S: survived; Yr: year. This table helps demonstrate that, while HME can occur in a wide range of ages, it is most often seen in older patients. Interestingly, PCR was most often used to help diagnose HME. Notably, most patients received doxycycline and had a good outcome.

it was associated with more severe disease $[8,10,23]$. Transplant type has been associated with worse outcomes and more prolonged ICU admissions, with lung transplant being associated with a poorer prognosis compared with other solid organ transplants $[10,24]$. The case fatality rate has been reported between $0 \%$ and $26 \%$ for transplant patients, which is similar to the case fatality rate in all HME cases [8-10]. Case fatality has decreased since its discovery despite the number of cases increasing [1].

This case report adds to the body of literature on ehrlichiosis in transplant patients, serves as an example of clinical patterns of infection, and stresses the importance of early identification of disease to enable prompt treatment within the vulnerable population of transplant recipients.

\section{Data Availability}

The data used to support the findings of this study are included within the article.

\section{Conflicts of Interest}

The authors declare that they have no conflicts of interest.

\section{Acknowledgments}

We would like to show appreciation to the Methodist University Hospital and the University of Tennessee Health Science Center.

\section{References}

[1] CDC, "Ehrlichiosis," 2021, Available from: https://www.cdc gov/ehrlichiosis/index.html.

[2] E. Rojero-Vázquez, G. Gordillo-Pérez, and M. Weber, "Infection of Anaplasma phagocytophilum and Ehrlichia spp. in opossums and dogs in Campeche, Mexico: the role of tick infestation," Frontiers in Ecology and Evolution, vol. 5, no. $161,2017$. 
[3] I. Rochlin and A. Toledo, "Emerging tick-borne pathogens of public health importance: a mini-review," Journal of Medical Microbiology, vol. 69, no. 6, p. 781, 2020.

[4] B. Doudier, J. Olano, P. Parola, and P. Brouqui, "Factors contributing to emergence of Ehrlichia and Anaplasma spp. as human pathogens," Veterinary Parasitology, vol. 167, no. 2-4, pp. 149-154, 2010.

[5] L. S. Muraro, A. D. O. Souza, T. N. Leite et al., "First evidence of Ehrlichia minasensis infection in horses from Brazil," Pathogens, vol. 10, no. 3, p. 265, 2021.

[6] M. J. Yabsley, "Natural history of Ehrlichia chaffeensis: vertebrate hosts and tick vectors from the United States and evidence for endemic transmission in other countries," Veterinary Parasitology, vol. 167, no. 2-4, pp. 136-148, 2010.

[7] E. M. Masterson, S. Gupta, N. Jakharia, and J. E. Peacock Jr., "Ehrlichiosis in a recent kidney transplant recipient: the repellent that did not repel! A case report and literature review of ehrlichiosis in solid organ transplant patients," Transplant Infectious Disease, vol. 22, no. 4, 2020.

[8] K. Kuriakose, A. C. Pettit, J. Schmitz, A. Moncayo, and K. C. Bloch, "Assessment of risk factors and outcomes of severe ehrlichiosis infection," JAMA Network Open, vol. 3, no. 11, p. e2025577, 2020.

[9] L. Thomas, I. Hongo, K. C. Bloch, Y. W. Tang, and S. Dummer, "Human ehrlichiosis in transplant recipients," American Journal of Transplantation, vol. 7, no. 6, pp. 16411647, 2007.

[10] K. Lawrence, M. R. Morrell, G. A. Storch, R. R. Hachem, and E. P. Trulock, "Clinical outcomes of solid organ transplant recipients with ehrlichiosis," Transplant Infectious Disease, vol. 11, no. 3, pp. 203-210, 2009.

[11] Z. K. Otrock, C. S. Eby, and C.-A. D. Burnham, "Human ehrlichiosis at a tertiary-care academic medical center: clinical associations and outcomes of transplant patients and patients with hemophagocytic lymphohistiocytosis," Blood Cells, Molecules, and Diseases, vol. 77, pp. 17-22, 2019.

[12] C. Radcliffe, C. Tsay, K. Glerum, J. Liao, G. Goshua, and G. Kerins, "Fever, pancytopenia, and elevated D-dimer in a 95-year-old woman with ehrlichiosis: a case report," $B M C$ Geriatrics, vol. 21, no. 1, pp. 1-4, 2021.

[13] P. Brouqui, F. Bacellar, G. Baranton et al., "Guidelines for the diagnosis of tick-borne bacterial diseases in Europe," Clinical Microbiology and Infection, vol. 10, no. 12, pp. 1108-1132, 2004.

[14] H. P. Tan, J. S. Dumler, W. R. Maley et al., "Human monocytic ehrlichiosis: an emerging pathogen in transplantation," Transplantation, vol. 71, no. 11, pp. 1678-1680, 2001.

[15] A. Saha, C. Browning, R. Dandamudi et al., "Donor-derived ehrlichiosis: 2 clusters following solid organ transplantation," Clinical Infectious Diseases, 2021.

[16] M. E. Reller and J. S. Dumler, "Development and clinical validation of a multiplex real-time quantitative PCR assay for human infection by Anaplasma phagocytophilum and Ehrlichia chaffeensis," Tropical medicine and infectious disease, vol. 3, no. 1, p. 14, 2018.

[17] K. S. Hamilton, S. M. Standaert, and M. C. Kinney, "Characteristic peripheral blood findings in human ehrlichiosis," Modern Pathology, vol. 17, no. 5, pp. 512-517, 2004.

[18] H. Regunath, C. Rojas-Moreno, J. P. Olano, R. D. Hammer, and W. Salzer, "Early diagnosis ofEhrlichia ewin- giiinfection in a lung transplant recipient by peripheral blood smear," Transplant Infectious Disease, vol. 19, no. 2, 2017.

[19] Z. K. Otrock, M. D. Gonzalez, and C. S. Eby, "Ehrlichiainduced hemophagocytic lymphohistiocytosis: a case series and review of literature," Blood Cells, Molecules, and Diseases, vol. 55, no. 3, pp. 191-193, 2015.

[20] C. A. Bell and R. Patel, "A real-time combined polymerase chain reaction assay for the rapid detection and differentiation of Anaplasma phagocytophilum, Ehrlichia chaffeensis, and Ehrlichia ewingii," Diagnostic Microbiology and Infectious Disease, vol. 53, no. 4, pp. 301-306, 2005.

[21] K. Abusaada, S. Ajmal, and L. Hughes, "Successful treatment of human monocytic ehrlichiosis with rifampin," Cureus, vol. 8 , no. $1,2016$.

[22] T. Fazili, E. Bansal, D. Garner, V. Bajwa, H. Kaur, and C. Schleupner, "Ehrlichia chaffeensis-associated hemophagocytic lymphohistiocytosis: a case series and literature review," American Journal of Medical Case Reports, vol. 9, no. 11, pp. 557-563, 2021.

[23] N. Safdar, R. B. Love, and D. G. Maki, "Severe Ehrlichia chaffeensis infection in a lung transplant recipient: a review of ehrlichiosis in the immunocompromised patient," Emerging Infectious Diseases, vol. 8, no. 3, p. 320, 2002.

[24] A. M. Liddell, J. W. Sumner, C. D. Paddock et al., "Reinfection with Ehrlichia chaffeensis in a liver transplant recipient," Clinical Infectious Diseases, vol. 34, no. 12, pp. 1644-1647, 2002.

[25] S. H. Sachdev, V. Joshi, E. R. Cox, A. Amoroso, and S. Palekar, "Severe life-threateningEhrlichia chaffeensisinfections transmitted through solid organ transplantation," Transplant Infectious Disease, vol. 16, no. 1, pp. 119-124, 2014.

[26] N. Kumar, J. Goyal, A. Goel, B. Shakoory, and W. Chatham, "Macrophage activation syndrome secondary to human monocytic ehrlichiosis," Indian Journal of Hematology and Blood Transfusion, vol. 30, Supplement 1, pp. 145$147,2014$.

[27] C. Cotant, J. F. Okulicz, B. Brezina, D. J. Riley, and N. G. Conger, "Human monocytic ehrlichiosis in a renal transplant patient," Scandinavian Journal of Infectious Diseases, vol. 38, no. 8, pp. 699-702, 2006.

[28] R. S. Buller, M. Arens, S. P. Hmiel et al., "Ehrlichia ewingii, a newly recognized agent of human ehrlichiosis," The New England Journal of Medicine, vol. 341, no. 3, pp. 148-155, 1999.

[29] R. Sadikot, M. J. Shaver, and W. B. Reeves, "Ehrlichia chaffeensis in a renal transplant recipient," American Journal of Nephrology, vol. 19, no. 6, pp. 674-676, 1999.

[30] G. E. Schutze and R. F. Jacobs, "Human monocytic ehrlichiosis in children," Pediatrics, vol. 100, no. 1, p. e10, 1997.

[31] H. Dorn, B. Dickinson, A. Agarwal, and K. L. Brayman, "Human ehrlichiosis after treatment of acute cellular rejection in a kidney transplant Patient," Transplantation, vol. 94, no. 10 S, p. 531, 2012.

[32] S. J. Antony, J. S. Dummer, and E. Hunter, "Human ehrlichiosis in a liver transplant recipient," Transplantation, vol. 60, no. 8, pp. 879-880, 1995.

[33] J. Trofe, K. S. Reddy, R. J. Stratta et al., "Human granulocytic ehrlichiosis in pancreas transplant recipients," Transplant Infectious Disease, vol. 3, no. 1, pp. 34-39, 2001. 
[34] J. A. Adachi, E. M. Grimm, P. Johnson, M. Uthman, B. Kaplan, and R. M. Rakita, "Human granulocytic ehrlichiosis in a renal transplant PATIENT," Transplantation, vol. 64, no. 8, pp. 1139-1142, 1997.

[35] M. D. Vannorsdall, S. Thomas, R. P. Smith et al., "Human granulocytic ehrlichiosis in a renal allograft recipient: review of the clinical spectrum of disease in solid organ transplant patients," Transplant Infectious Disease, vol. 4, no. 2, pp. 97$101,2002$.

[36] W. Hassan, M. Talwar, V. Balaraman, and M. Z. Molnar, "Ehrlichiosis infection mimicking thrombotic microangiopathy syndrome early after kidney transplantation," Transplant Infectious Disease, vol. 22, no. 5, 2020. 